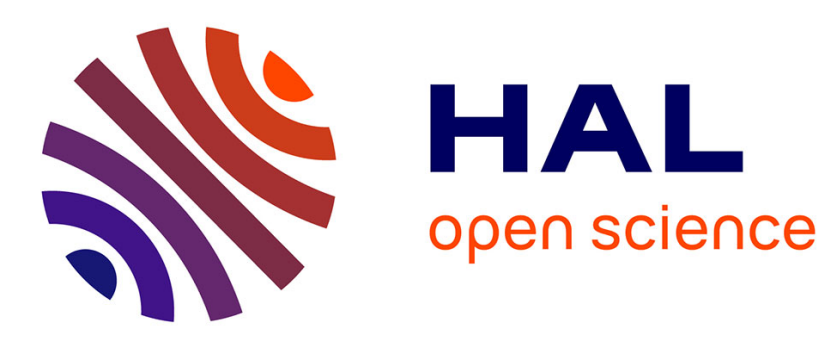

\title{
Ionic-Liquid-Supported Synthesis of Amines and Derivatives
}

Mansour Debdab, Florence Mongin, J.P. Bazureau

\section{To cite this version:}

Mansour Debdab, Florence Mongin, J.P. Bazureau. Ionic-Liquid-Supported Synthesis of Amines and Derivatives. Synthesis: Journal of Synthetic Organic Chemistry, 2006, 23, pp.4046-4052. 10.1055/s2006-950309 . hal-00772538

\section{HAL Id: hal-00772538 https://hal.science/hal-00772538}

Submitted on 11 Jan 2013

HAL is a multi-disciplinary open access archive for the deposit and dissemination of scientific research documents, whether they are published or not. The documents may come from teaching and research institutions in France or abroad, or from public or private research centers.
L'archive ouverte pluridisciplinaire HAL, est destinée au dépôt et à la diffusion de documents scientifiques de niveau recherche, publiés ou non, émanant des établissements d'enseignement et de recherche français ou étrangers, des laboratoires publics ou privés. 


\title{
Ionic Liquid Supported Synthesis of Amines and Derivatives.
}

\author{
Mansour Debdab, ${ }^{\mathrm{a}, \mathrm{b}}$ Florence Mongin, ${ }^{\mathrm{a}, *}$ Jean Pierre Bazureau ${ }^{\mathrm{a}, \mathrm{b}, *}$ \\ ${ }^{a}$ Synthèse \& ElectroSynthèse Organiques, UMR 6510, CNRS, Bâtiment 10A, Université de Rennes 1, Campus de Beaulieu, Avenue du \\ Général Leclerc, 35042 Rennes Cedex, France \\ ${ }^{b}$ Present address: Sciences Chimiques de Rennes, UMR 6226, CNRS, Bâtiment 10A, Université de Rennes 1, Campus de Beaulieu, \\ Avenue du Général Leclerc, 35042 Rennes Cedex, France \\ Fax: +33(0)223236374 \\ E-mail: florence.mongin@univ-rennes1.fr, jean-pierre.bazureau@univ-rennes1.fr \\ Received: The date will be inserted once the manuscript is accepted.
}

\begin{abstract}
Amine precursors such as NH-Boc (5) and NH-formyl (6) protected glycines were grafted by esterification on the hydroxylated arms of 1-(2-hydroxyethyl)-3-methylimidazolium hexafluorophosphates $\mathbf{4 h}$ or tetrafluoroborates $\mathbf{4} \boldsymbol{t}$. The Boc cleavage was then realized at room temperature by successively treating acetonitrile solutions of the carbamates $7 \boldsymbol{h}$ or $7 \boldsymbol{t}$ with methanol and acetyl chloride ( 2 equivalents each). Interestingly, the hydrochlorides were converted to the corresponding amines $9 \boldsymbol{h}$ or $9 \boldsymbol{t}$, respectively, during the removal of the solvent. Ugi reaction of the ionic liquid-grafted amine $9 \mathrm{~b} t$ with phthalaldehydic acid and tertbutylisonitrile, followed by cleavage, furnished the phthalimidine 12.
\end{abstract}

Key words: ionic liquids, amines, glycine derivatives, esters, Ugi reaction

\section{Introduction}

The demand for increasing numbers of compounds, notably with potential therapeutic value, caused chemists to look for ways to simplify, expedite, and automate the process of organic molecules syntheses. Since Merrifield introduced the solid-phase peptide synthesis, ${ }^{1}$ insoluble supports rapidly developed into a means for facile purification processes, with easier phase separation and purification to remove excess reagents and side products, and possible automation. More recently alternative methodologies appeared in order to compensate for the drawbacks related to the heterogeneous nature of the insoluble polymers. Soluble polymer supports were aimed at restoring homogeneous reaction conditions although retaining the easy purification of the product; ${ }^{2}$ however, limitations such as a low loading capacity can restrict their applications. The use of fluorous phase synthesis proved efficient for the separation of small molecules, ${ }^{3}$ but the expense of perfluoroalkane solvents limits its development. In 2001, low molecular weight ionic liquids were used as soluble supports for organic synthesis. ${ }^{4}$

Ionic liquids, notably those including 1-alkyl-3methylimidazolium cations, have been largely used in organic synthesis as reaction media. ${ }^{5} \mathrm{~A}$ feature of ionic liquids is that their solubilities in organic and aqueous phases can be tuned by varying the nature of the anion and ring substituents. Taking advantage of this opportunity, labs including ours studied and demonstrated the efficiency of ionic liquid supported syntheses. ${ }^{4,6}$ As a continuation of our work on functionalized ionic liquids, we have synthesized a series of amines that could be involved in multicomponent reactions utilizing isonitriles.

Few syntheses of amino ionic liquids are described in the literature. ${ }^{6 e, 7}$ The amines we here describe are connected to the imidazolium parts with an ester function that could be cleaved to recycle the ionic support. Moreover, the substitution at $\mathrm{C} 2$ of the azolium ring was also considered for possible use in reactions with bases. ${ }^{8}$

\section{Results and discussion}

The synthesis of the 2-substituted 1-methylimidazoles $\mathbf{2 a}$ and $\mathbf{b}$ from commercially available 2-ethylimidazole (1a) and 2-phenylimidazole (1b), respectively, was effected by improving a method described in the literature. ${ }^{9}$ The use of iodomethane ( 2 equiv) in basic media under phase transfer catalysis afforded the expected methylated compound $\mathbf{2 a}$ in $36 \%$ yield, due to the competitive formation of 2-ethyl-1,3-dimethylimidazolium iodide. Using a stoichiometric amount of iodomethane avoided the side-product to be formed, and afforded $\mathbf{2 a}$ in $89 \%$ yield. The compound $\mathbf{2 b}$ was similarly obtained in $97 \%$ yield. Quaternarisation of the 2-substituted imidazoles 2a-c was carried out as described for the compound $\mathbf{2 d},{ }^{10}$ by simply heating the substrate with 2chloroethanol at $120^{\circ} \mathrm{C}$. Except for the compound $\mathbf{3 b}$, which proved to be too viscous and was directly involved in the next step, the chlorides $\mathbf{3}$ were easily purified by washing with diethyl ether and drying. Anion metathesis using either potassium hexafluorophosphate or ammonium tetrafluoroborate was effected as reported for the compound 3d. ${ }^{10}$ Filtration of ammonium or potassium chloride, which are insoluble in acetonitrile, afforded the hexafluorophosphates $\mathbf{4 a} \boldsymbol{h}, \mathbf{4 b} \boldsymbol{h}, \mathbf{4} \boldsymbol{c h}$ and $\mathbf{4 d} \boldsymbol{h}$, and the tetrafluoroborates $4 \mathbf{c} \boldsymbol{t}$ and $\mathbf{4 d} \boldsymbol{t}$ in excellent yields (Scheme 1). 


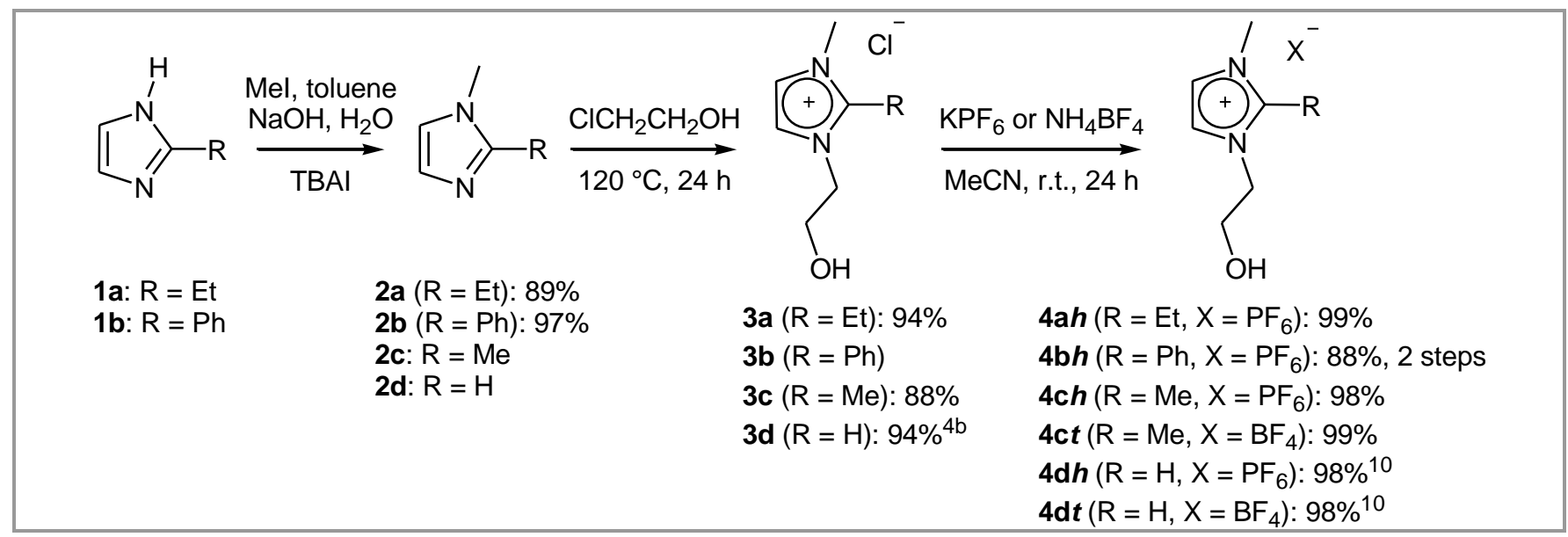

Scheme 1

NH-Boc and NH-formyl glycines were chosen to be grafted on the hydroxylated arm of synthesized ionic liquids. The esterification of the protected aminoacids $\mathbf{5 , 6}$ was effected with the ionic liquids $\mathbf{4 c , d}$ in dry acetonitrile in the presence of dicyclohexylcarbodiimide (DCC) and a catalytic amount of 4-(N,Ndimethylamino)pyridine (DMAP), by adapting a procedure described. ${ }^{4 a}$ Dicyclohexylurea was easily removed by filtration, and washings of the crude residue with ethyl acetate or diethyl ether were efficient to afford the pure expected imidazolium salts $\mathbf{7 , 8}$ in yields ranging from 74 to $96 \%$ (Scheme 2). On the other hand, the ionic liquid $\mathbf{4 b h}$ proved to be a bad support due to its good solubility in ethyl acetate or diethyl ether, which does not allow washings.

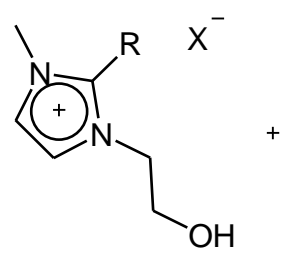<smiles>O=C(O)CN[Pb]</smiles>

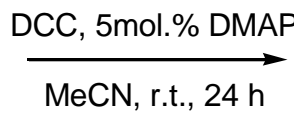

\begin{abstract}
4ch: $\mathrm{R}=\mathrm{Me}, \mathrm{X}=\mathrm{PF}_{6}$
4ct: $\mathrm{R}=\mathrm{Me}, \mathrm{X}=\mathrm{BF}_{4}$

4d $h: R=H, X=P F_{6}$
\end{abstract}

4dt: $\mathrm{R}=\mathrm{H}, \mathrm{X}=\mathrm{BF}_{4}$

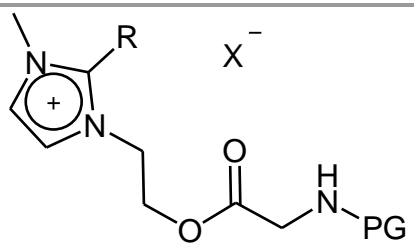

7ah $\left(R=M e, X=P F_{6}, P G=B o c\right): 96 \%$ $7 \mathrm{~b} \boldsymbol{h}\left(\mathrm{R}=\mathrm{H}, \mathrm{X}=\mathrm{PF}_{6}, \mathrm{PG}=\mathrm{Boc}\right): 92 \%$

7 at $\left(R=M e, X=B_{4}, P G=B o c\right): 90 \%$

$7 b \boldsymbol{t}\left(\mathrm{R}=\mathrm{H}, \mathrm{X}=\mathrm{BF}_{4}, \mathrm{PG}=\mathrm{Boc}\right): 92 \%$

$8 \mathrm{a} h\left(\mathrm{R}=\mathrm{Me}, \mathrm{X}=\mathrm{PF}_{6}, \mathrm{PG}=\mathrm{CHO}\right): 74 \%$

$8 b \boldsymbol{h}\left(\mathrm{R}=\mathrm{H}, \mathrm{X}=\mathrm{PF}_{6}, \mathrm{PG}=\mathrm{CHO}\right): 76 \%$

8at $\left(\mathrm{R}=\mathrm{Me}, \mathrm{X}=\mathrm{BF}_{4}, \mathrm{PG}=\mathrm{CHO}\right): 80 \%$

$8 b t\left(R=H, X=B_{4}, P G=C H O\right): 96 \%$

\section{Scheme 2}

The cleavage of Boc-protected amines 7 was next investigated. The use of trifluoroacetic acid in dichloromethane is often efficient for this purpose, but can cause a partial ionic liquid anion exchange with trifluoroacetate. We thus preferred to use hydrochloric acid in an organic solvent. ${ }^{6 e}$ Surprisingly, by treating acetonitrile solutions of the carbamates 7 with excess hydrochloric acid in ethyl acetate, ${ }^{11}$ the amines were straight away obtained after the evaporation of acetonitrile instead of the attempted hydrochlorides, avoiding a subsequent neutralisation step. ${ }^{12}$ The optimisation of the reactions was next carried out in NMR tubes by successively treat- ing solutions of the carbamates 7 in $\mathrm{CD}_{3} \mathrm{CN}$ with methanol ( 1 equiv) and acetyl chloride ( 1 equiv). The monitoring of the reaction at room temperature showed that there was no more change after $24 \mathrm{~h}$, and that the expected hydrochlorides were formed in about $50 \%$ conversion rates. Adding a second equiv of methanol and acetyl chloride to the NMR tubes led to the hydrochlorides in $100 \%$ conversions. Removal of the solvent from the reaction mixtures, washings and drying afforded the corresponding amines $\mathbf{9}$, as verified by mass spectrometry, in good yields (Scheme 3). 


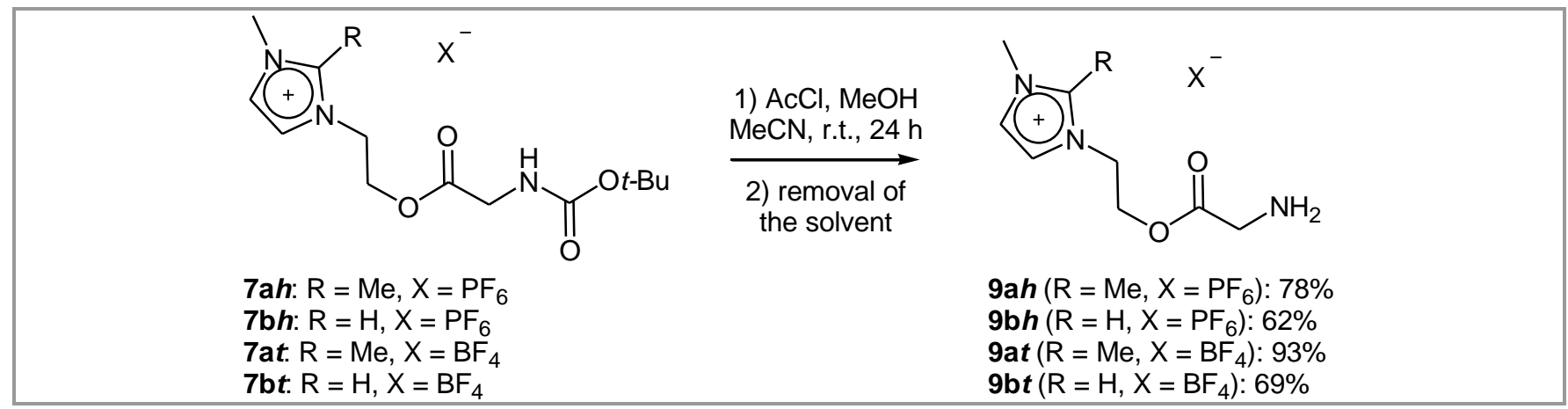

Scheme 3

Since the Boc cleavage was found to work satisfactorily, we did not study the formyl cleavage of $\mathbf{8}$. We rather decided to involve the amines $\mathbf{9}$ in a multicomponent reaction utilizing isonitriles.

Among the multi-component transformations using amines, the Ugi four-component reaction (U-4CR) which involves amine, aldehyde, carboxylic acid and isonitrile, is one of the most frequently employed process. ${ }^{13}$

Preliminary studies in order to compare the different ionic supports were realized by treating the $O$-gratfed glycines 9 with 4-chlorobenzaldehyde, 2-nitrobenzoic acid and tert-butylisonitrile in methanol at room tem- perature or $40{ }^{\circ} \mathrm{C} .{ }^{14}$ Even if the Ugi compounds were partially cleaved from the ionic support during the reactions, it could be noted that the imidazolium ring (substituted at $\mathrm{C} 2$ or not) was compatible with tertbutylisonitrile under the conditions used. ${ }^{15}$ Moreover, the Ugi reactions were found to be more efficient using the borate anion.

The subsequent treatment of partially cleaved Ugi compounds $\mathbf{1 0} t$ with triethylamine ${ }^{16}$ in methanol at room temperature furnished the corresponding methyl ester 11; the latter was identified by characteristic NMR peaks and mass spectrometry, but was insufficiently stable to be purified (Scheme 4).<smiles>C=NC(C)(C)C</smiles>

\section{Scheme 4}

On the other hand, the reaction between the amine 9bt, phthalaldehydic acid and tert-butylisonitrile, ${ }^{17}$ followed by the cleavage from the ionic support, furnished a stable compound. Indeed, the ester 12 could be easily purified by chromatography over silica gel and crystallisation (Scheme 5, Route A).

In order to avoid partial cleavage during the Ugi reaction, we decided to use a solvent different from methanol. The Ugi reaction was performed in acetoni- trile. The solubility of the amine $\mathbf{9 b} t$ in acetonitrile is very low but using a long reaction time ( 5 days), we could obtain the $O$-grafted ester $\mathbf{1 3}$ in a $56 \%$ yield without observing cleavage or degradation. The unreacted amine $9 \mathrm{~b} t$ could be easily removed by filtration, giving after evaporation of acetonitrile and washings with diethyl ether the attempted Ugi product 13. Subsequent cleavage under the conditions used before finally afforded the compound $\mathbf{1 2}$ in a $80 \%$ yield (Scheme 5, Route B). 


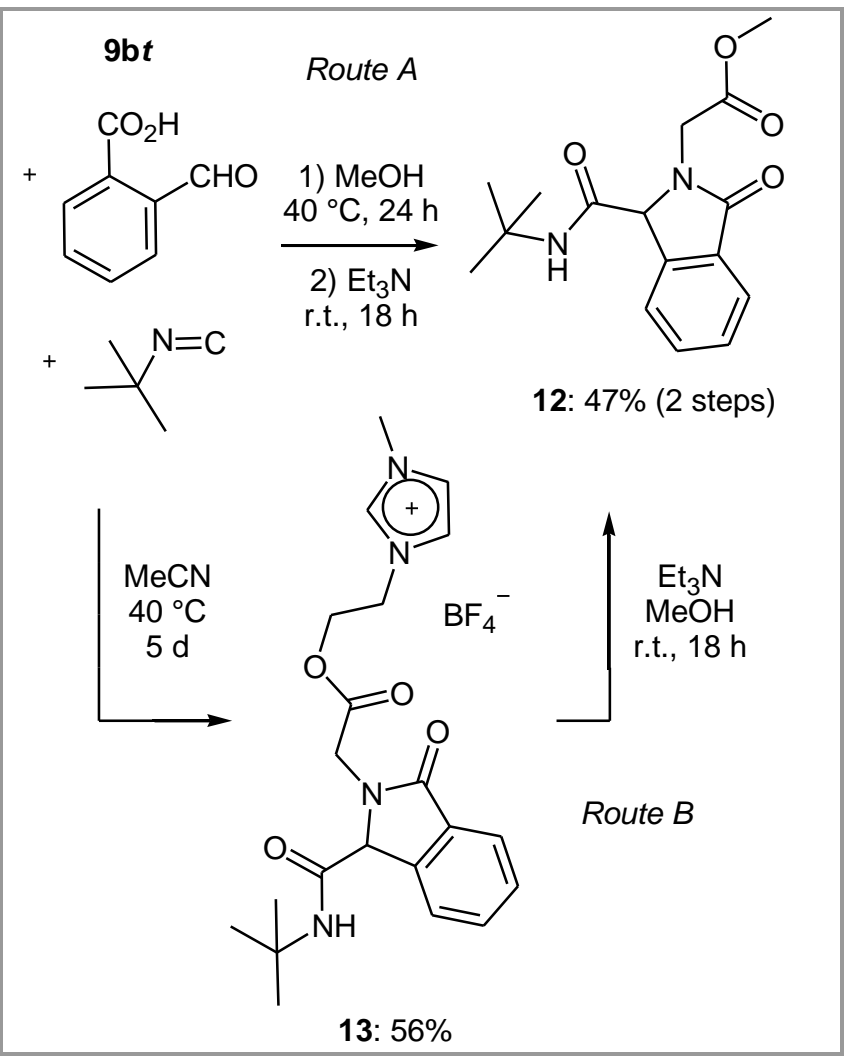

Scheme 5

\section{Conclusion}

We have shown that amines can be very easily prepared from the corresponding NH-Boc using ionic liquids as

The ${ }^{1} \mathrm{H}$ and ${ }^{13} \mathrm{C}$ NMR spectra were recorded at 200 and $50 \mathrm{MHz}$, respectively, on a Bruker ARX $200 \mathrm{P}$ spectrometer. $N$-Formylglycine (6) was prepared by adapting a procedure described. ${ }^{18}$ The ionic liquids $\mathbf{3 d},{ }^{4 \mathrm{~b}} \mathbf{4} \mathbf{d h}{ }^{10}$ and $\mathbf{4 d t} \mathbf{t}^{10}$ were prepared according to literature procedures.

\section{2-Ethyl-1-methylimidazole (2a). ${ }^{19}$}

To a biphasic mixture obtained from 2-ethylimidazole (1a, $9.8 \mathrm{~g}, 0.10 \mathrm{~mol})$, tetrabutylammonium iodide (1.9 $\mathrm{g}, 5.1 \mathrm{mmol}), 50 \%$ aqueous $\mathrm{NaOH}(350 \mathrm{~mL})$ and toluene $(280 \mathrm{~mL})$, was added iodomethane $(7.1 \mathrm{~mL}, 0.11$ mol). After stirring for $15 \mathrm{~min}$ at r.t., the mixture was diluted with toluene $(280 \mathrm{~mL})$ and water $(280 \mathrm{~mL})$. The organic phase was separated, dried over $\mathrm{MgSO}_{4}$, and concentrated under reduced pressure.

Pale yellow oil; yield: $9.8 \mathrm{~g}(89 \%)$.

IR (KBr): 3390, 2977, 2940, 1529, 1501, 1470, 1416, $1283,1146,1059,730,623 \mathrm{~cm}^{-1}$. supports. This ionic liquid supported synthesis involving amines or derivatives offers the advantage of simple products isolations using washings with appropriate solvents; moreover, NMR monitorings of reactions proved to be easily feasible. The imidazolium-type ionic liquids $O$-bound glycine derivatives thus prepared can be used in Ugi reaction.

${ }^{1} \mathrm{H}$ NMR $\left(\mathrm{CDCl}_{3}\right) \delta=6.90(\mathrm{~d}, J=1.1 \mathrm{~Hz}, 1 \mathrm{H}), 6.77$ $(\mathrm{d}, J=1.1 \mathrm{~Hz}, 1 \mathrm{H}), 3.55(\mathrm{~s}, 3 \mathrm{H}), 2.66(\mathrm{q}, J=7.5 \mathrm{~Hz}$, $2 \mathrm{H}), 1.31$ (t, $J=7.6 \mathrm{~Hz}, 3 \mathrm{H}$ ).

${ }^{13} \mathrm{C} \mathrm{NMR}\left(\mathrm{CDCl}_{3}\right) \delta=147.5,124.8,118.7,30.5,18.2$, 10.1 .

Anal. Calcd for $\mathrm{C}_{6} \mathrm{H}_{10} \mathrm{~N}_{2}$ (110.16): C, 65.42; H, 9.15; N, 25.43. Found: C, 65.31; H, 9.32; N, 25.21.

\section{1-Methyl-2-phenylimidazole (2b).}

To a biphasic mixture obtained from 2phenylimidazole (1b, $10 \mathrm{~g}, 69 \mathrm{mmol})$, tetrabutylammonium iodide $(1.9 \mathrm{~g}, 5.1 \mathrm{mmol}), 50 \%$ aqueous $\mathrm{NaOH}(350 \mathrm{~mL})$ and toluene $(280 \mathrm{~mL})$, was added iodomethane $(4.9 \mathrm{~mL}, 76 \mathrm{mmol})$. After stirring for 15 min at r.t., the mixture was diluted with toluene $(280$ $\mathrm{mL})$ and water $(280 \mathrm{~mL})$. The organic phase was separated, dried over $\mathrm{MgSO}_{4}$, and concentrated under reduced pressure.

Pale yellow oil; yield: $11 \mathrm{~g} \mathrm{(97 \% )}$.

Anal. Calcd for $\mathrm{C}_{10} \mathrm{H}_{10} \mathrm{~N}_{2}$ (158.20): C, 75.92; H, 6.37; N, 17.71. Found: C, 75.68; H, 6.52; N, 17.44. 
The spectroscopic data were identical to those previously described. ${ }^{20}$

\section{2-Ethyl-1-(2-hydroxyethyl)-3-methylimidazolium chloride (3a).}

A mixture of 2-chloroethanol $(0.67 \mathrm{~mL}, 10 \mathrm{mmol})$ and 2-ethyl-1-methylimidazole (2a, $1.1 \mathrm{~g}, 10 \mathrm{mmol})$ was heated for $24 \mathrm{~h}$ at $120^{\circ} \mathrm{C}$. The crude product, which crystallized upon cooling, was finely crushed, washed with diethyl ether $(3 \times 80 \mathrm{~mL})$, and dried under reduced pressure for $10 \mathrm{~h}$.

Pale yellow powder; $\mathrm{mp} 55-58{ }^{\circ} \mathrm{C}$; yield: $1.8 \mathrm{~g}(94 \%)$. ${ }^{1} \mathrm{H}$ NMR (DMSO- $\left.d_{6}\right) \delta=7.66(\mathrm{~s}, 2 \mathrm{H}), 6.79(\mathrm{~d}, J=$ $1.1 \mathrm{~Hz}, 1 \mathrm{H}), 5.25(\mathrm{br} \mathrm{s}, 1 \mathrm{H}), 4.20(\mathrm{t}, J=4.9 \mathrm{~Hz}, 2$ H), $3.82(\mathrm{~s}, 3 \mathrm{H}), 3.71(\mathrm{t}, J=7,7 \mathrm{~Hz}, 2 \mathrm{H}), 3.06$ (q, $J=$ 7,6 Hz, $2 \mathrm{H}), 1.19$ (t, $J=7,6 \mathrm{~Hz}, 3 \mathrm{H})$.

${ }^{13} \mathrm{C}$ NMR (DMSO- $\left.d_{6}\right) \delta=148.2(\mathrm{q}), 122.6(\mathrm{t}), 121.3$ (t), $59.6(\mathrm{~s}), 50.1(\mathrm{~s}), 34.6(\mathrm{p}), 16.1(\mathrm{~s}), 11.0(\mathrm{p})$.

HRMS: $m / z \mathrm{C}^{+}$calcd for $\mathrm{C}_{8} \mathrm{H}_{15} \mathrm{~N}_{2} \mathrm{O}$ : 155.1184 ; found: 155.1176 .

\section{1-(2-Hydroxyethyl)-2,3-dimethylimidazolium chlo- ride $(3 b)$.}

The procedure is as described for $\mathbf{3 a}$ but using 1methyl-2-phenylimidazole $(\mathbf{2 b}, 1.6 \mathrm{~g}, 10 \mathrm{mmol})$ instead of $\mathbf{2 a}$. Compound $\mathbf{3 b}$ being too viscous to consider its washing, it was involved in the next step without purification.

\section{1-(2-Hydroxyethyl)-2,3-dimethylimidazolium chlo- ride $(3 \mathrm{c})$.}

The procedure is as described for 3a but using 1,2dimethylimidazole $(0.96 \mathrm{~g}, 10 \mathrm{mmol})$ instead of $\mathbf{2 a}$.

Beige powder; $\mathrm{mp} 58^{\circ} \mathrm{C}$; yield: $1.6 \mathrm{~g}(88 \%)$.

${ }^{1} \mathrm{H}$ NMR (DMSO- $\left.d_{6}\right) \delta=7.59(\mathrm{~s}, 2 \mathrm{H}), 5.08(\mathrm{t}, J=5.1$ $\mathrm{Hz}, 1 \mathrm{H}), 4.17(\mathrm{t}, J=5.0 \mathrm{~Hz}, 2 \mathrm{H}), 3.75(\mathrm{~s}, 3 \mathrm{H}), 3.68$ (q, $J=4.8 \mathrm{~Hz}, 2 \mathrm{H}), 2.57$ (s, $3 \mathrm{H})$.

${ }^{13} \mathrm{C}$ NMR (DMSO- $\left.d_{6}\right) \delta=144.9,122.2,121.3,59.7$, 50.3, 34.8, 9.8.

HRMS: $m / z \quad \mathrm{C}^{+}$calcd for $\mathrm{C}_{7} \mathrm{H}_{13} \mathrm{~N}_{2} \mathrm{O}$ : 141.10279; found: 141.1030 .

\section{2-Ethyl-1-(2-hydroxyethyl)-3-methylimidazolium hexafluorophosphate $(4 \mathrm{a} h)$.}

A mixture of 3a $(11 \mathrm{~g}, 56 \mathrm{mmol})$ and potassium hexafluorophosphate $(10 \mathrm{~g}, 56 \mathrm{mmol})$ in $\mathrm{MeCN}(370 \mathrm{~mL})$ was stirred for $24 \mathrm{~h}$ at r.t. in a dry atmosphere. After filtration over celite, removal of the solvent, and drying under reduced pressure, the product was kept un$\operatorname{der} \mathrm{N}_{2}$.

Pale yellow powder; $\mathrm{mp}<50{ }^{\circ} \mathrm{C}$; yield: $17 \mathrm{~g}(99 \%)$. ${ }^{1} \mathrm{H}$ NMR (acetone- $\left.d_{6}\right) \delta=7.64(\mathrm{~d}, J=2.1 \mathrm{~Hz}, 1 \mathrm{H})$, $7.60(\mathrm{~d}, J=2.1 \mathrm{~Hz}, 1 \mathrm{H}), 4.41(\mathrm{t}, J=4.9 \mathrm{~Hz}, 2 \mathrm{H})$, $4.00(\mathrm{~s}, 3 \mathrm{H}), 3.96$ (t, $J=5.0 \mathrm{~Hz}, 2 \mathrm{H}), 3.24$ (q, $J=7.7$ $\mathrm{Hz}, 2 \mathrm{H}), 1.37(\mathrm{t}, J=7.7 \mathrm{~Hz}, 3 \mathrm{H}), \mathrm{OH}$ not seen.

${ }^{13} \mathrm{C}$ NMR (DMSO- $d_{6}$ ) $\delta=148.2,122.6,121.3,59.6$, 50.1, 34.6, 16.2, 11.0.

HRMS: $m / z\left[2 \mathrm{C}^{+}, \mathrm{PF}_{6}^{-}\right]^{+}$calcd for $\mathrm{C}_{16} \mathrm{H}_{30} \mathrm{~N}_{4} \mathrm{O}_{2} \mathrm{~F}_{6} \mathrm{P}$ : 455.2011; found: 455.2011 .

\section{1-(2-Hydroxyethyl)-3-methyl-2-phenylimidazolium hexafluorophosphate $(4 \mathrm{~b} h)$.}

The procedure is as described for $\mathbf{4} \mathbf{a} \boldsymbol{h}$ but using $\mathbf{3 b}$ (13 g, $56 \mathrm{mmol})$ instead of $\mathbf{3 a}$.

Beige powder; $\mathrm{mp}<50{ }^{\circ} \mathrm{C}$; yield: $17 \mathrm{~g}(88 \%)$.

${ }^{1} \mathrm{H}$ NMR (acetone- $\left.d_{6}\right) \delta=7.91(\mathrm{~d}, J=2.0 \mathrm{~Hz}, 1 \mathrm{H})$, $7.8(\mathrm{~m}, 6 \mathrm{H}), 4.26(\mathrm{t}, J=5.0 \mathrm{~Hz}, 2 \mathrm{H}), 3.92(\mathrm{t}, J=5.1$ $\mathrm{Hz}, 2 \mathrm{H}), 3.87$ (s, $3 \mathrm{H}), \mathrm{OH}$ not seen.

${ }^{13} \mathrm{C}$ NMR (DMSO- $d_{6}$ ) $\delta=144.8,132.4,130.9,129.6$, 123.5, 122.1, 121.5, 59.4, 50.9, 35.6.

HRMS: $m / z \mathrm{C}^{+}$calcd for $\mathrm{C}_{12} \mathrm{H}_{15} \mathrm{~N}_{2} \mathrm{O}$ : 203.11844; found: 203.1184 .

1-(2-Hydroxyethyl)-2,3-dimethylimidazolium hexafluorophosphate $(4 \mathrm{ch})$.

The procedure is as described for $\mathbf{4 a} \boldsymbol{h}$ but using $\mathbf{3 c}$ (9.9 g, $56 \mathrm{mmol})$ instead of 3a.

Pale brown oil; yield: $16 \mathrm{~g}$ (98\%).

${ }^{1} \mathrm{H}$ NMR (acetone- $\left.d_{6}\right) \delta=7.60(\mathrm{~s}, 1 \mathrm{H}), 7.58(\mathrm{~s}, 1 \mathrm{H})$, 4.39 (t, $J=4.6 \mathrm{~Hz}, 2 \mathrm{H}), 3.97(\mathrm{~m}, 6 \mathrm{H}), 2.77(\mathrm{~s}, 3 \mathrm{H})$.

${ }^{13} \mathrm{C}$ NMR (acetone- $\left.d_{6}\right) \delta=145.9,122.8,121.8,60.9$, 51.1, 35.0, 9.4.

HRMS: $m / z\left[2 \mathrm{C}^{+}, \mathrm{PF}_{6}^{-}\right]^{+}$calcd for $\mathrm{C}_{14} \mathrm{H}_{26} \mathrm{~N}_{4} \mathrm{O}_{2} \mathrm{~F}_{6} \mathrm{P}$ : 427.16976; found: 427.1700 .

\section{1-(2-Hydroxyethyl)-2,3-dimethylimidazolium tetra- fluoroborate $(4 c t)$.}

A mixture of $3 \mathbf{c}(11 \mathrm{~g}, 56 \mathrm{mmol})$ and ammonium tetrafluoroborate $(5.9 \mathrm{~g}, 56 \mathrm{mmol})$ in $\mathrm{MeCN}(370 \mathrm{~mL})$ was stirred for $24 \mathrm{~h}$ at r.t. in a dry atmosphere. After filtration over celite, removal of the solvent, and drying under reduced pressure, the product was kept under $\mathrm{N}_{2}$.

Pale brown oil; yield: $13 \mathrm{~g}$ (99\%).

${ }^{1} \mathrm{H}$ NMR (DMSO- $\left.d_{6}\right) \delta=7.63(\mathrm{~s}, 2 \mathrm{H}), 5.25(\mathrm{t}, J=5.5$ $\mathrm{Hz}, 1 \mathrm{H}), 4.19(\mathrm{t}, J=5.0 \mathrm{~Hz}, 2 \mathrm{H}), 3.76(\mathrm{~s}, 3 \mathrm{H}), 3.67$ (q, $J=4.8 \mathrm{~Hz}, 2 \mathrm{H}), 2.59$ (s, $3 \mathrm{H})$.

${ }^{13} \mathrm{C}$ NMR (acetone- $\left.d_{6}\right) \delta=145.9,122.7,121.8,60.9$, 51.0, 35.0, 9.4.

HRMS: $m / z\left[2 \mathrm{C}^{+}, \mathrm{BF}_{4}\right]^{+}$calcd for $\mathrm{C}_{14} \mathrm{H}_{26} \mathrm{~N}_{4} \mathrm{O}_{2} \mathrm{~F}_{4}{ }^{11} \mathrm{~B}$ : 369.20849; found: 369.2091 .

1-[2-[(N-(tertbutoxycarbonyl)aminomethyl)carbonyloxy]ethyl]- 


\section{2,3-dimethylimidazolium (7ah). \\ hexafluorophosphate}

1,3-Dicyclohexylcarbodiimide $(0.89 \mathrm{~g}, 4.3 \mathrm{mmol})$ and 4-(dimethylamino)pyridine $(26 \mathrm{mg}, 0.21 \mathrm{mmol})$ were added to a solution of $\mathbf{4 c h}(1.2 \mathrm{~g}, 4.3 \mathrm{mmol})$ in $\mathrm{MeCN}$ $(30 \mathrm{~mL})$ at r.t. After $10 \mathrm{~min}, \quad \mathrm{~N}$-(tertbutoxycarbonyl)glycine $(\mathbf{5}, 0.79 \mathrm{~g}, 4.5 \mathrm{mmol})$ was introduced, and the mixture was stirred for $24 \mathrm{~h}$ at r.t. before filtration over celite, removal of the solvent, washing with diethyl ether $(2 \times 80 \mathrm{~mL})$ and $1: 1 \mathrm{AcO}-$ $\mathrm{Et} /$ pentane $(80 \mathrm{~mL})$, and drying under reduced pressure for $10 \mathrm{~h}$.

Beige powder; mp $120{ }^{\circ} \mathrm{C}$; yield: $1.8 \mathrm{~g}(96 \%)$.

${ }^{1} \mathrm{H}$ NMR (acetone- $\left.d_{6}\right) \delta=7.71(\mathrm{~d}, J=2.2 \mathrm{~Hz}, 1 \mathrm{H})$, $7.64(\mathrm{~d}, J=2.1 \mathrm{~Hz}, 1 \mathrm{H}), 6.40(\mathrm{br} \mathrm{s}, 1 \mathrm{H}), 4.67$ (t, $J=$ $4.6 \mathrm{~Hz}, 2 \mathrm{H}), 4.55(\mathrm{t}, J=4.5 \mathrm{~Hz}, 2 \mathrm{H}), 3.98(\mathrm{~s}, 3 \mathrm{H})$, $3.82(\mathrm{~d}, J=6.2 \mathrm{~Hz}, 2 \mathrm{H}), 2.85(\mathrm{~s}, 3 \mathrm{H}), 1.40(\mathrm{~s}, 9 \mathrm{H})$.

${ }^{13} \mathrm{C}$ NMR (acetone- $\left.d_{6}\right) \delta=170.8,156.9,146.5,123.5$, 122.3, 79.5, 63.4, 48.0, 42.7, 35.6, 28.5, 10.0.

HRMS: $m / z \mathrm{C}^{+}$calcd for $\mathrm{C}_{14} \mathrm{H}_{24} \mathrm{~N}_{3} \mathrm{O}_{4}$ : 298.17668; found: 298.1764 .

\section{1-[2-[(N-(tert-}

butoxycarbonyl)aminomethyl)carbonyloxy]ethyl]3-methylimidazolium hexafluorophosphate $(7 \mathrm{bh})$.

The procedure is as described for $7 \mathbf{a} h$ but using $\mathbf{4 d} \boldsymbol{h}$ (1.2 g, $4.3 \mathrm{mmol})$ instead of $\mathbf{4} \boldsymbol{c h}$.

White powder; $\mathrm{mp} 70{ }^{\circ} \mathrm{C}$; yield: $1.7 \mathrm{~g}(92 \%)$.

${ }^{1} \mathrm{H}$ NMR (acetone- $\left.d_{6}\right) \delta=9.13(\mathrm{~s}, 1 \mathrm{H}), 7.85(\mathrm{~s}, 1 \mathrm{H})$, $7.76(\mathrm{~s}, 1 \mathrm{H}), 6.46(\mathrm{br} \mathrm{s}, 1 \mathrm{H}), 4.70(\mathrm{t}, J=4.7 \mathrm{~Hz}, 2$ $\mathrm{H}), 4.61(\mathrm{t}, J=4.7 \mathrm{~Hz}, 2 \mathrm{H}), 4.11(\mathrm{~s}, 3 \mathrm{H}), 3.86(\mathrm{~d}, J=$ $6.2 \mathrm{~Hz}, 2 \mathrm{H}), 1.41(\mathrm{~s}, 9 \mathrm{H})$.

${ }^{13} \mathrm{C}$ NMR (acetone- $\left.d_{6}\right) \delta=170.8,157.0,138.0,124.7$, 123.8, 79.5, 63.2, 49.5, 42.7, 36.7, 28.5.

HRMS: $m / z\left[2 \mathrm{C}^{+}, \mathrm{PF}_{6}^{-}\right]^{+}$calcd for $\mathrm{C}_{26} \mathrm{H}_{44} \mathrm{~N}_{6} \mathrm{O}_{8} \mathrm{~F}_{6} \mathrm{P}$ : 713.28625; found: 713.2862.

\section{1-[2-[(N-(tert-}

butoxycarbonyl)aminomethyl)carbonyloxy]ethyl]2,3-dimethylimidazolium tetrafluoroborate (7at).

The procedure is as described for $\mathbf{7} \mathbf{a} \boldsymbol{h}$ but using $\mathbf{4} \boldsymbol{c} t$ (0.98 g, $4.3 \mathrm{mmol})$ instead of $\mathbf{4} \mathbf{c h}$.

White powder; mp $128^{\circ} \mathrm{C}$; yield: $1.5 \mathrm{~g}(90 \%)$.

${ }^{1} \mathrm{H}$ NMR (acetone- $\left.d_{6}\right) \delta=7.68(\mathrm{~d}, J=2.0 \mathrm{~Hz}, 1 \mathrm{H})$, $7.63(\mathrm{~d}, J=1.9 \mathrm{~Hz}, 1 \mathrm{H}), 6.36(\mathrm{br} \mathrm{s}, 1 \mathrm{H}), 4.63$ (t, $J=$ $4.5 \mathrm{~Hz}, 2 \mathrm{H}), 4.55(\mathrm{t}, J=4.5 \mathrm{~Hz}, 2 \mathrm{H}), 3.95(\mathrm{~s}, 3 \mathrm{H})$, $3.81(\mathrm{~d}, J=6.2 \mathrm{~Hz}, 2 \mathrm{H}), 2.82(\mathrm{~s}, 3 \mathrm{H}), 1.41(\mathrm{~s}, 9 \mathrm{H})$.

${ }^{13} \mathrm{C}$ NMR (acetone- $\left.d_{6}\right) \delta=170.8,156.9,146.5,123.5$, 122.2, 79.4, 63.5, 47.9, 42.7, 35.5, 28.5, 9.8.

HRMS: $m / z \mathrm{C}^{+}$calcd for $\mathrm{C}_{14} \mathrm{H}_{24} \mathrm{~N}_{3} \mathrm{O}_{4}$ : 298.17668; found: 298.1771 .

\section{$1-[2-[(N-(t e r t-$}

butoxycarbonyl)aminomethyl)carbonyloxy]ethyl]3-methylimidazolium tetrafluoroborate $(7 \mathrm{~b} t)$.

The procedure is as described for $\mathbf{7 a} \boldsymbol{h}$ but using $\mathbf{4 d} \boldsymbol{t}$ $(0.92 \mathrm{~g}, 4.3 \mathrm{mmol})$ instead of $\mathbf{4 c h}$.

White powder; mp $95{ }^{\circ} \mathrm{C}$; yield: $1.5 \mathrm{~g}(92 \%)$.

${ }^{1} \mathrm{H}$ NMR (acetone- $\left.d_{6}\right) \delta=9.10(\mathrm{~s}, 1 \mathrm{H}), 7.83(\mathrm{~s}, 1 \mathrm{H})$, $7.74(\mathrm{~s}, 1 \mathrm{H}), 6.41(\mathrm{br} \mathrm{s}, 1 \mathrm{H}), 4.67(\mathrm{t}, J=4.7 \mathrm{~Hz}, 2$ H), 4.59 (t, $J=4.7 \mathrm{~Hz}, 2 \mathrm{H}), 4.08(\mathrm{~s}, 3 \mathrm{H}), 3.85$ (d, $J=$ $6.2 \mathrm{~Hz}, 2 \mathrm{H}), 1.40(\mathrm{~s}, 9 \mathrm{H})$.

${ }^{13} \mathrm{C}$ NMR (acetone- $\left.d_{6}\right) \delta=170.9,156.9,138.2,124.7$, 123.8, 79.4, 63.3, 49.5, 43.8, 36.6, 28.5.

HRMS: $m / z \mathrm{C}^{+}$calcd for $\mathrm{C}_{13} \mathrm{H}_{22} \mathrm{~N}_{3} \mathrm{O}_{4}$ : 284.16103; found: 284.1613 .

\section{1-[2-[(Formylaminomethyl)carbonyloxy]ethyl]-2,3- dimethylimidazolium hexafluorophosphate (8ah).}

The procedure is as described for $7 \mathbf{a} \boldsymbol{h}$ but using $N$ formylglycine $(\mathbf{6}, 0.46 \mathrm{~g}, 4.5 \mathrm{mmol})$ instead of $\mathbf{5}$, and washing with AcOEt $(3 \times 50 \mathrm{~mL})$.

Beige powder; mp $116{ }^{\circ} \mathrm{C}$; yield: $1.2 \mathrm{~g}(74 \%)$.

${ }^{1} \mathrm{H}$ NMR (DMSO- $d_{6}$ ) $\delta=8.45$ (br s, $1 \mathrm{H}$ ), 8.08 (s, 1 H), $7.64(\mathrm{~s}, 1 \mathrm{H}) 7.63(\mathrm{~s}, 1 \mathrm{H}), 4.40(\mathrm{~s}, 4 \mathrm{H}), 3.86(\mathrm{~d}, J$ $=6.2 \mathrm{~Hz}, 2 \mathrm{H}), 3.75(\mathrm{~s}, 3 \mathrm{H}), 2.60(\mathrm{~s}, 3 \mathrm{H})$.

${ }^{13} \mathrm{C}$ NMR (DMSO- $d_{6}$ ) $\delta=169.3,161.9,145.2,122.5$, 121.3, 62.8, 46.5, 39.4, 34.8, 9.3.

HRMS: $m / z \mathrm{C}^{+}$calcd for $\mathrm{C}_{10} \mathrm{H}_{16} \mathrm{~N}_{3} \mathrm{O}_{3}$ : 226.11917; found: 226.1188 .

\section{1-[2-[(Formylaminomethyl)carbonyloxy]ethyl]-3- methylimidazolium hexafluorophosphate $(8 \mathrm{bh})$.}

The procedure is as described for $7 \mathbf{a} \boldsymbol{h}$ but using $\mathbf{4 d} \boldsymbol{h}$ $(1.2 \mathrm{~g}, 4.3 \mathrm{mmol})$ instead of $\mathbf{4 c h}, N$-formylglycine (6, $0.46 \mathrm{~g}, 4.5 \mathrm{mmol}$ ) instead of $\mathbf{5}$, and washing with AcOEt $(3 \times 50 \mathrm{~mL})$.

Pale brown oil; yield: $1.2 \mathrm{~g} \mathrm{(76 \% ).}$

${ }^{1} \mathrm{H}$ NMR (acetone- $\left.d_{6}\right) \delta=9.06(\mathrm{~s}, 1 \mathrm{H}), 8.21(\mathrm{~s}, 1 \mathrm{H})$, $7.81(\mathrm{~s}, 1 \mathrm{H}), 7.70(\mathrm{~s}, 1 \mathrm{H}), 7.64(\mathrm{br} \mathrm{s}, 1 \mathrm{H}), 4.70(\mathrm{t}, J$ $=4.4 \mathrm{~Hz}, 2 \mathrm{H}), 4.58(\mathrm{t}, J=4.5 \mathrm{~Hz}, 2 \mathrm{H}), 4.07(\mathrm{~s}, 3 \mathrm{H})$, $4.02(\mathrm{~d}, J=6.1 \mathrm{~Hz}, 2 \mathrm{H})$.

${ }^{13} \mathrm{C}$ NMR (acetone- $\left.d_{6}\right) \delta=170.0,162.7,138.0,124.7$, 123.7, 63.5, 49.4, 40.3, 36.6.

HRMS: $m / z \mathrm{C}^{+}$calcd for $\mathrm{C}_{9} \mathrm{H}_{14} \mathrm{~N}_{3} \mathrm{O}_{3}: 212.1035$; found: 212.1036.

1-[2-[(Formylaminomethyl)carbonyloxy]ethyl]-2,3dimethylimidazolium tetrafluoroborate (8at).

The procedure is as described for $\mathbf{7} \mathbf{a} \boldsymbol{h}$ but using $\mathbf{4 c t}$ $(0.98 \mathrm{~g}, 4.3 \mathrm{mmol})$ instead of $\mathbf{4} \boldsymbol{c h}, N$-formylglycine $(\mathbf{6}, 0.46 \mathrm{~g}, 4.5 \mathrm{mmol})$ instead of $\mathbf{5}$, and washing with AcOEt $(3 \times 50 \mathrm{~mL})$.

White powder; $\mathrm{mp} 54{ }^{\circ} \mathrm{C}$; yield: $1.1 \mathrm{~g}(80 \%)$. 
${ }^{1} \mathrm{H}$ NMR $\left(\right.$ DMSO- $\left.d_{6}\right) \delta=8.44(\mathrm{br} \mathrm{s}, 1 \mathrm{H}), 8.08(\mathrm{~s}, 1$ $\mathrm{H}), 7.64(\mathrm{~s}, 1 \mathrm{H}) 7.63(\mathrm{~s}, 1 \mathrm{H}), 4.41(\mathrm{~s}, 4 \mathrm{H}), 3.86(\mathrm{~d}, J$ $=6.0 \mathrm{~Hz}, 2 \mathrm{H}), 3.75(\mathrm{~s}, 3 \mathrm{H}), 2.63(\mathrm{~s}, 3 \mathrm{H})$.

${ }^{13} \mathrm{C}$ NMR (DMSO- $\left.d_{6}\right) \delta=169.3,161.9,145.1,122.5$, 121.3, 62.8, 46.6, 39.4, 34.8, 9.3.

HRMS: $m / z \mathrm{C}^{+}$calcd for $\mathrm{C}_{10} \mathrm{H}_{16} \mathrm{~N}_{3} \mathrm{O}_{3}$ : 226.1192; found: 226.1188 .

1-[2-[(Formylaminomethyl)carbonyloxy]ethyl]-3methylimidazolium tetrafluoroborate $(8 \mathrm{~b} t)$.

The procedure is as described for $\mathbf{7 a} \boldsymbol{h}$ but using $\mathbf{4 d} t$ $(0.92 \mathrm{~g}, 4.3 \mathrm{mmol})$ instead of $\mathbf{4} \boldsymbol{c h}, \mathrm{N}$-formylglycine $(\mathbf{6}, 0.46 \mathrm{~g}, 4.5 \mathrm{mmol})$ instead of $\mathbf{5}$, and washing with $\operatorname{AcOEt}(3 \times 50 \mathrm{~mL})$.

Pale brown oil; yield: $1.2 \mathrm{~g}(96 \%)$.

${ }^{1} \mathrm{H}$ NMR (acetone- $\left.d_{6}\right) \delta=9.04(\mathrm{~s}, 1 \mathrm{H}), 8.20(\mathrm{~s}, 1 \mathrm{H})$, $7.81(\mathrm{~s}, 1 \mathrm{H}), 7.70(\mathrm{~s}, 1 \mathrm{H}), 7.61(\mathrm{br} \mathrm{s}, 1 \mathrm{H}), 4.65(\mathrm{t}, J$ $=4.6 \mathrm{~Hz}, 2 \mathrm{H}), 4.55(\mathrm{t}, J=4.4 \mathrm{~Hz}, 2 \mathrm{H}), 4.05(\mathrm{~s}, 3 \mathrm{H})$, $4.01(\mathrm{~d}, J=6.1 \mathrm{~Hz}, 2 \mathrm{H})$.

${ }^{13} \mathrm{C}$ NMR (acetone- $\left.d_{6}\right) \delta=170.0,162.7,140.1,124.7$, 123.7, 63.7, 49.3, 40.3, 36.6.

HRMS: $m / z \mathrm{C}^{+}$calcd for $\mathrm{C}_{9} \mathrm{H}_{14} \mathrm{~N}_{3} \mathrm{O}_{3}:$ 212.10352; found: 212.1040 .

\section{1-[2-[(Aminomethyl)carbonyloxy]ethyl]-2,3-} dimethylimidazolium hexafluorophosphate (9ah).

A solution of $7 \mathbf{a} h(1.1 \mathrm{~g}, 2.5 \mathrm{mmol})$ in acetonitrile (5 $\mathrm{mL})$ was successively treated with methanol $(0.20$ $\mathrm{mL}, 5.0 \mathrm{mmol})$ and acetyl chloride $(0.36 \mathrm{~mL}, 5.0$ mmol). After $24 \mathrm{~h}$ at r.t., the solvent was removed under reduced pressure. The residue was washed with AcOEt $(2 \times 10 \mathrm{~mL})$ and dried under reduced pressure for $10 \mathrm{~h}$.

Beige powder; $\mathrm{mp} 130{ }^{\circ} \mathrm{C}$; yield: $0.67 \mathrm{~g}(78 \%)$.

${ }^{1} \mathrm{H}$ NMR (DMSO- $\left.d_{6}\right) \delta=8.54$ (br s, $\left.2 \mathrm{H}\right), 7.73(\mathrm{~d}, J=$ $1.9 \mathrm{~Hz}, 1 \mathrm{H}), 7.66(\mathrm{~d}, J=1.9 \mathrm{~Hz}, 1 \mathrm{H}), 4.46(\mathrm{~s}, 4 \mathrm{H})$, 3.79 (s, $2 \mathrm{H}), 3.77$ (s, $3 \mathrm{H}), 2.61$ (s, $3 \mathrm{H})$.

${ }^{13} \mathrm{C}$ NMR (DMSO- $d_{6}$ ) $\delta=167.2,145.2,122.6,121.4$, 63.7, 46.5, 39.8, 34.9, 9.6.

HRMS: $m / z \mathrm{C}^{+}$calcd for $\mathrm{C}_{9} \mathrm{H}_{16} \mathrm{~N}_{3} \mathrm{O}_{2}:$ 198.12425; found: 198.1245 .

\section{1-[2-[(Aminomethyl)carbonyloxy]ethyl]-3-} methylimidazolium hexafluorophosphate $(9 \mathrm{~b} h)$.

The procedure is as described for $\mathbf{9 a} \boldsymbol{h}$ but using $\mathbf{7 b} \boldsymbol{h}$ $(1.1 \mathrm{~g}, 2.5 \mathrm{mmol})$ instead of $\mathbf{7} \mathbf{a}$.

Beige powder; $\mathrm{mp} 153{ }^{\circ} \mathrm{C}$; yield: $0.51 \mathrm{~g}(62 \%)$.

${ }^{1} \mathrm{H}$ NMR (DMSO- $\left.d_{6}\right) \delta=9.24(\mathrm{~s}, 1 \mathrm{H}), 8.25(\mathrm{br} \mathrm{s}, 2$ $\mathrm{H}), 7.81(\mathrm{~s}, 1 \mathrm{H}), 7.73(\mathrm{~s}, 1 \mathrm{H}), 4.49(\mathrm{~s}, 4 \mathrm{H}), 3.87(\mathrm{~s}, 3$ $\mathrm{H}), 3.80$ (s, $2 \mathrm{H})$.

${ }^{13} \mathrm{C}$ NMR (acetone- $d_{6}$ ) $\delta=167.0,137.3,123.5,122.6$, $63.5,47.8,39.6,35.8$.
HRMS: $m / z \mathrm{C}^{+}$calcd for $\mathrm{C}_{8} \mathrm{H}_{14} \mathrm{~N}_{3} \mathrm{O}_{2}$ : 184.10860; found: 184.1087 .

1-[2-[(Aminomethyl)carbonyloxy]ethyl]-2,3dimethylimidazolium tetrafluoroborate (9at).

The procedure is as described for $\mathbf{9} \mathbf{a} \boldsymbol{h}$ but using $\mathbf{7 a}$ $(0.96 \mathrm{~g}, 2.5 \mathrm{mmol})$ instead of $7 \mathbf{a} \boldsymbol{h}$.

White powder; mp $159{ }^{\circ} \mathrm{C}$; yield: $0.66 \mathrm{~g}$ (93\%).

${ }^{1} \mathrm{H}$ NMR (DMSO- $\left.d_{6}\right) \delta=8.44$ (br s, $2 \mathrm{H}$ ), 7.73 (s, 1 H), $7.65(\mathrm{~s}, 1 \mathrm{H}), 4.46(\mathrm{~s}, 4 \mathrm{H}), 3.79(\mathrm{~s}, 2 \mathrm{H}), 3.76(\mathrm{~s}, 3$ $\mathrm{H}), 2.61(\mathrm{~s}, 3 \mathrm{H})$.

${ }^{13} \mathrm{C}$ NMR (DMSO- $d_{6}$ ) $\delta=167.3,145.2,122.5,121.4$, 63.7, 46.4, 39.8, 34.9, 9.6.

HRMS: $m / z \mathrm{C}^{+}$calcd for $\mathrm{C}_{9} \mathrm{H}_{16} \mathrm{~N}_{3} \mathrm{O}_{2}: 198.1243$; found: 198.1245 .

1-[2-[(Aminomethyl)carbonyloxy]ethyl]-3methylimidazolium tetrafluoroborate $(9 \mathrm{~b} t)$.

The procedure is as described for $9 \mathbf{a} h$ but using $7 \mathbf{b} t$ $(0.93 \mathrm{~g}, 2.5 \mathrm{mmol})$ instead of $7 \mathbf{a} \boldsymbol{h}$.

White powder; $\mathrm{mp} 125-126{ }^{\circ} \mathrm{C}$; yield: $0.47 \mathrm{~g}(69 \%)$.

${ }^{1} \mathrm{H}$ NMR (DMSO- $\left.d_{6}\right) \delta=9.26(\mathrm{~s}, 1 \mathrm{H}), 8.41$ (br s, 2 $\mathrm{H}), 7.82(\mathrm{~s}, 1 \mathrm{H}), 7.73(\mathrm{~s}, 1 \mathrm{H}), 4.50(\mathrm{~s}, 4 \mathrm{H}), 3.87(\mathrm{~s}, 3$ $\mathrm{H}), 3.82(\mathrm{~s}, 2 \mathrm{H})$.

${ }^{13} \mathrm{C}$ NMR (DMSO- $\left.d_{6}\right) \delta=167.1,137.4,123.6,122.7$, 63.6, 47.9, 39.7, 35.9.

HRMS: $m / z \mathrm{C}^{+}$calcd for $\mathrm{C}_{8} \mathrm{H}_{14} \mathrm{~N}_{3} \mathrm{O}_{2}$ : 184.10860; found: 184.1087 .

\section{1-[2-[[N-[1-(4-chlorophenyl)-2-[(1,1-} dimethylethyl)amino]-2-oxoethyl $]-N-[(2-$ nitro-

phenyl)carbonyl]aminomethyl]carbonyloxy]ethyl]2,3-dimethylimidazolium tetrafluoroborate (10at).

4-Chlorobenzaldehyde $(0.16 \mathrm{~g}, 1.1 \mathrm{mmol})$ was added to a solution of $9 \mathrm{a} t(0.29 \mathrm{~g}, 1.0 \mathrm{mmol})$ in methanol (2 $\mathrm{mL})$. After $10 \mathrm{~min}$ at $40^{\circ} \mathrm{C}, 2$-nitrobenzoic acid $(0.19$ $\mathrm{g}, 1.1 \mathrm{mmol})$ and tert-butylisonitrile $(127 \mu \mathrm{L}, 1.1$ mmol) were added to the mixture in a dry atmosphere. After $24 \mathrm{~h}$ at $40{ }^{\circ} \mathrm{C}$, the solvent was removed under reduced pressure, the residue was washed with diethyl ether $(2 \times 10 \mathrm{~mL})$ and dissolved in acetonitrile $(4 \mathrm{~mL})$. After filtration and evaporation of the filtrate, the residue was dried under reduced pressure for $10 \mathrm{~h}$.

HRMS: $\mathrm{m} / \mathrm{z} \mathrm{C}^{+}$calcd for $\mathrm{C}_{28} \mathrm{H}_{33} \mathrm{~N}_{5} \mathrm{O}_{6}{ }^{35} \mathrm{Cl}$ : 570.21194; found: 570.2117 .

1-[2-[[N-[1-(4-chlorophenyl)-2-[(1,1dimethylethyl)amino]-2-oxoethyl]- $N-[(2-$ nitro-

phenyl)carbonyl]aminomethyl]carbonyloxy]ethyl]3-methylimidazolium tetrafluoroborate (10bt). 
The procedure is as described for 10at but using $9 \mathbf{b} t$ $(0.27 \mathrm{~g}, 1.0 \mathrm{mmol})$ instead of 9at.

HRMS: $\mathrm{m} / \mathrm{z} \mathrm{C}^{+}$calcd for $\mathrm{C}_{27} \mathrm{H}_{31} \mathrm{~N}_{5} \mathrm{O}_{6}{ }^{35} \mathrm{Cl}$ : 556.19629; found: 556.1968 .

\section{1-[2-[[N-[3-(tert-butylaminocarbonyl)] phthalimidino]carbonyloxy]ethyl]-3- methylimidazolium tetrafluoroborate (13).}

Phthalaldehydic acid $(0.17 \mathrm{~g}, 1.1 \mathrm{mmol})$ was added to a solution of $9 \mathrm{~b} t(0.27 \mathrm{~g}, 1.0 \mathrm{mmol})$ in $\mathrm{MeCN}(4 \mathrm{~mL})$. After $20 \mathrm{~min}$ at $40^{\circ} \mathrm{C}$, tert-butylisonitrile $(127 \mu \mathrm{L}, 1.1$ mmol) was added to the mixture. After $5 \mathrm{~d}$ at $40{ }^{\circ} \mathrm{C}$, the mixture was filtered in order to remove the unreacted amine. The solvent was then removed under reduced pressure, and the residue was washed with diethyl ether $(2 \times 10 \mathrm{~mL})$ and dried under reduced pressure for $10 \mathrm{~h}$.

Pale brown oil; yield: $0.27 \mathrm{~g}(56 \%)$.

${ }^{1} \mathrm{H}$ NMR (acetone- $\left.d_{6}\right) \delta=9.04(\mathrm{~s}, 1 \mathrm{H}), 8.08(\mathrm{~s}, 1 \mathrm{H})$, $8.07(\mathrm{~s}, 1 \mathrm{H}), 7.7(\mathrm{~m}, 4 \mathrm{H}), 6.72(\mathrm{~s}, 1 \mathrm{H}), 5.29(\mathrm{~s}, 1 \mathrm{H})$, $4.6(\mathrm{~m}, 3 \mathrm{H}), 4.0(\mathrm{~m}, 6 \mathrm{H}), 1.31(\mathrm{~s}, 9 \mathrm{H})$.

${ }^{13} \mathrm{C}$ NMR (acetone- $d_{6}$ ) $\delta=169.3,168.5,166.4,142.9$, 137.6, 134.9, 131.3, 131.2, 125.1, 124.2, 124.0, 123.1, 65.1, 63.4, 51.5, 48.7, 43.5, 36.0, 26.9 (3C).

HRMS: $m / z \quad \mathrm{C}^{+}$calcd for $\mathrm{C}_{21} \mathrm{H}_{27} \mathrm{~N}_{4} \mathrm{O}_{4}$ : 399.2032; found: 399.2033 .

\section{Methyl [N-[3-(tert-butylaminocarbonyl)] phthalimidino]acetate (12).}

\section{Route A.}

Phthalaldehydic acid $(0.17 \mathrm{~g}, 1.1 \mathrm{mmol})$ was added to a solution of $9 \mathrm{~b} t(0.27 \mathrm{~g}, 1.0 \mathrm{mmol})$ in methanol (2 $\mathrm{mL})$. After $20 \mathrm{~min}$ at $40^{\circ} \mathrm{C}$, tert-butylisonitrile $(127$ $\mu \mathrm{L}, 1.1 \mathrm{mmol}$ ) was added to the mixture in a dry atmosphere. After $24 \mathrm{~h}$ at $40{ }^{\circ} \mathrm{C}$, the mixture was cooled to r.t. and triethylamine $(0.21 \mu \mathrm{L}, 1.5 \mathrm{mmol})$ was added. After stirring for $18 \mathrm{~h}$ at r.t., removal of the solvent, addition of water $(5 \mathrm{~mL})$, extraction with AcOEt, drying over $\mathrm{MgSO}_{4}$, and evaporation of the solvent, the crude product was chromatographed over silica gel using AcOEt/pentane (1/1) as an eluent and crystallized from diethyl ether.

\section{Route $B$.}

A solution of $13(0.49 \mathrm{~g}, 1 \mathrm{mmol})$ in methanol $(3 \mathrm{~mL})$ was treated with triethylamine $(0.21 \mu \mathrm{L}, 1.5 \mathrm{mmol})$, and stirred for $18 \mathrm{~h}$ at r.t. in a dry atmosphere. After removal of the solvent, addition of water $(5 \mathrm{~mL})$, extraction with AcOEt, drying over $\mathrm{MgSO}_{4}$, and evaporation of the solvent, the crude product was chromatographed over silica gel using AcOEt/pentane (1/1) as an eluent.

Beige powder; mp $148-150{ }^{\circ} \mathrm{C}$; yield: $0.14 \mathrm{~g}(47 \%, 2$ steps) using route $\mathrm{A}$ and $0.24 \mathrm{~g}(80 \%)$ using route $\mathrm{B}$.
${ }^{1} \mathrm{H}$ NMR (acetone- $\left.d_{6}\right) \delta=7.7(\mathrm{~m}, 5 \mathrm{H}), 5.22(\mathrm{~s}, 1 \mathrm{H})$, $4.68(\mathrm{~d}, J=18 \mathrm{~Hz}, 1 \mathrm{H}), 4.01(\mathrm{~d}, J=18 \mathrm{~Hz}, 1 \mathrm{H}), 3.74$ (s, $3 \mathrm{H}), 1.31$ (s, $9 \mathrm{H})$.

${ }^{13} \mathrm{C}$ NMR (acetone- $\left.d_{6}\right) \delta=170.7,169.6,166.8,143.2$, $132.9,131.6,129.4,123.3,104.0,65.7,52.5,51.9$, 43.7, 28.3 (3C).

Anal. Calcd for $\mathrm{C}_{16} \mathrm{H}_{20} \mathrm{~N}_{2} \mathrm{O}_{4}$ (304.14): C, 63.14; H, 6.62; N, 9.20. Found: C, 63.29; H, 6.64; N, 9.08.

\section{Acknowledgment}

We are indebted to Ministère de l'Enseignement Supérieur et de la Recherche scientifique de la République Algérienne Démocratique et Populaire (Coopération et échanges inter-universitaires, number 14/PG/FR./2005/2006) for financial support.

We thank Aurélien Bucher for his contribution to this study.

\section{References}

(1) Merrifield, R. B. J. Am. Chem. Soc. 1963, 85, 2149.

(2) (a) Gravert, D. J.; Janda, K. D. Chem. Rev. 1997, 97, 489. (b) Toy, P. H.; Janda, K. D. Acc. Chem. Res. 2000, 33, 546.

(3) Horvath, I. T. Acc. Chem. Res. 1998, 31, 641.

(4) (a) Fraga-Dubreuil, J.; Bazureau, J. P. Tetrahedron Lett. 2001, 42, 6097. (b) Fraga-Dubreuil, J.; Bazureau, J. P. Tetrahedron 2003, 59, 6121 .

(5) (a) Welton, T. Chem. Rev. 1999, 99, 2071. (b) Holbrey, D.; Seddon, K. R. Clean Prod. Process 1999, 1, 223. (c) Wasserscheid, P.; Keim, W. Angew. Chem., Int. Ed. 2000, 39, 3772. (d) Dupont, J.; de Souza, R. F.; Suarez, P. A. Z. Chem. Rev. 2002, 102, 3667.

(6) See for example: (a) Handy, S. T.; Okello, M. Tetrahedron Lett. 2003, 44, 8399; (b) Miao, W.; Chan, T. H. Org. Lett. 2003, 5, 5003; (c) Anjaiah, S.; Chandrasekhar, S.; Grée, R. Tetrahedron Lett. 2004, 45, 569; (d) de Kort, M.; Tuin, A. W.; Kuiper, S.; Overkleeft, H. S.; van der Marel, G. A.; Buijsman, R. C. Tetrahedron Lett. 2004, 45, 2171; (e) Miao, W.; Chan, T.-H. J. Org. Chem. 2005, 70, 3251; (f) Yi, F.; Peng, Y.; Song, G. Tetrahedron Lett. 2005, 46, 3931.

(7) See for example: (a) Mi, X.; Luo, S.; Cheng, J.-P. J. Org Chem. 2005, 70, 2338; (b) Song, G.; Cai, Y.; Peng, Y. J. Comb. Chem. 2005, 7, 561 .

(8) Concerning the reactivity of the imidazolium rings, see for example: (a) Nair, V.; Bindu, S.; Sreekumar, V. Angew. Chem. Int. Ed. 2004, 43, 5130; (b) Handy, S. T.; Okello, M. J. Org. Chem. 2005, 70, 1915.

(9) Casaschi, A.; Grigg, R.; Sansano, J. M. Tetrahedron 2001, 57, 607.

(10) Fraga-Dubreuil, J.; Famelart, M.-H.; Bazureau, J. P. Org. Proc. Res. \& Dev. 2002, 6, 374.

(11) The solution was prepared by mixing equimolar amounts of acetyl chloride and methanol in ethyl acetate: Nudelman, A.; Bechor, Y.; Falb, E.; Fischer, B.; Wexler, B. A.; Nudelman, A. Synth. Commun. 1998, 28, 471.

(12) Thermal dehydrochlorination from crystalline solids such as pyridinium salts of chlorometallates has been described: Adams, C. J.; Crawford, P. C.; Orpen, A. G.; Podesta, T. J.; Salt, B. Chem. Commun. 2005, 2457.

(13) Dömling, A.; Ugi, I. Angew. Chem. Int. Ed. 2000, 39, 3168.

(14) Ugi reactions are generally performed in methanol at room temperature or $40^{\circ} \mathrm{C}$ (see ref. 13).

(15) For a reaction example between a $\pi$-deficient aza-aromatic and an isonitrile, see: Ugi, I.; Böttner, E. Liebigs Ann. Chem. 1963, 670, 74. 
(16) Fantauzzi, P. P.; Yager, K. M. Tetrahedron Lett. 1998, 39, 1291.

(17) Concerning similar Ugi reaction between aliphatic amines, phthalaldehydic acid and tert-butylisonitrile, see: HanuschKompa, C.; Ugi, I. Tetrahedron Lett. 1998, 39, 2725.

(18) Fayol, A.; Housseman, C.; Sun, X.; Janvier, P.; Bienaymé, H.; Zhu, J. Synthesis 2005, 161.

(19) Misaki, T.; Nagase, R.; Matsumoto, K.; Tanabe, Y. J. Am. Chem. Soc. 2005, 127, 2854.

(20) Prasad, A. S. B.; Stevenson, T. M.; Citineni, J. R.; Nyzam, V.; Knochel, P. Tetrahedron 1997, 53, 7237. 
Ionic Liquid Supported Synthesis of Amines.
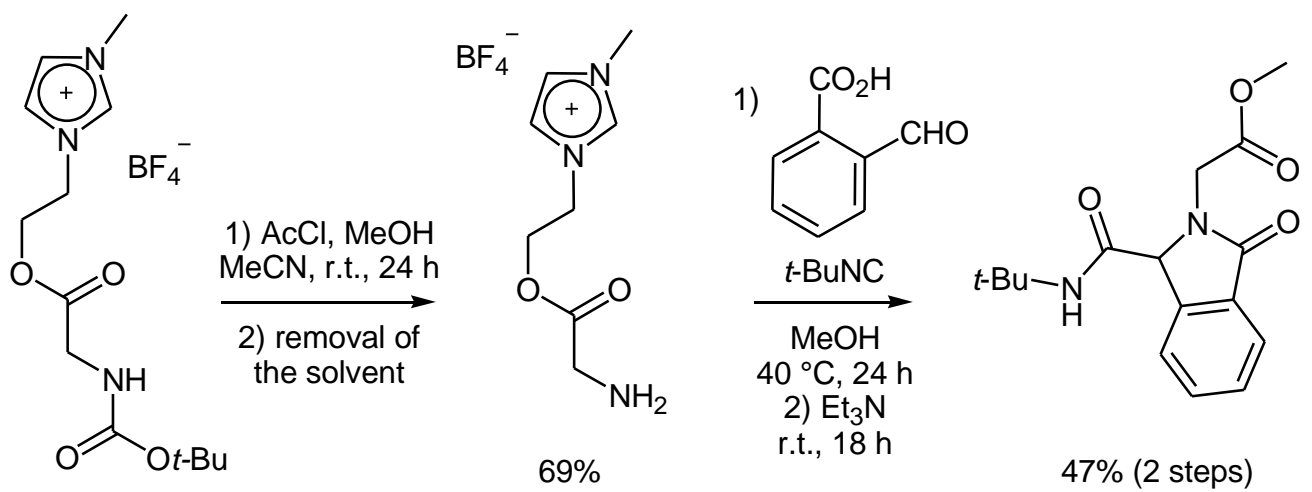